\title{
Intraoperative real-time three-dimensional transesophageal echocardiography as a precise navigator for a successful complicated postoperative left ventricular pseudoaneurysm repair: a case report
}

\author{
Futaba Miyoshi, Yusuke Seino ${ }^{*}$ (D), Minoru Nomura and Makoto Ozaki
}

\begin{abstract}
Background: Left ventricular pseudoaneurysm (LV-PAN) formation is a rare complication after cardiac surgery and mainly occurs after mitral valve surgery. Echocardiography plays a critical role in the assessment of rupture location, orifice geometry, and anatomical relationship with surrounding structures.

Case presentation: A 56-year-old man presented with LV-PAN formation 1 year after aortic root replacement combined with aortic replacement despite the lack of direct manipulation of the rupture site in the procedure and postoperative myocardial infarction. Intraoperative real-time three-dimensional transesophageal echocardiography (RT 3-D TEE) during surgical repair of the LV-PAN facilitated understanding of the shape of the LV-PAN orifice and the exact anatomical relationship between the rupture site and the posteromedial papillary muscle. Information sharing with surgeons contributed to avoiding direct papillary muscle injury and thus mitral valve deformation.

Conclusion: LV-PAN formation after cardiac surgery can present without direct manipulation of the rupture site and major coronary lesion. Intraoperative RT 3-D TEE can facilitate better understanding of the anatomical relationship between the rupture site and the posteromedial papillary muscle and allow for information sharing to avoid complications during surgical repair.
\end{abstract}

Keywords: Left ventricular pseudoaneurysm, Aortic root replacement, Real-time three-dimensional transesophageal echocardiography

\section{Background}

Left ventricular (LV) pseudoaneurysm (PAN) formation is a rare complication of cardiac operations and mainly occurs as a result of LV free wall rupture after mitral valve surgery $[1,2]$. The common causes of LV-PAN formation are transmural myocardial infarction, LV trauma, infection, and prior cardiac surgery, mainly mitral valve replacement; $33-58 \%$ of the LV-PAN cases results from cardiac surgery [1-4]. The PAN location depends on the operative procedure; for example, the PAN is formed in the posterior subannular region of the mitral valve after

\footnotetext{
* Correspondence: yu-seino@twmu.ac.jp

Department of Anesthesiology, Tokyo Women's Medical University, 8-1 Kawada-cho, Shinjuku-ku, Tokyo, Japan
}

mitral surgery [2]. The treatment of LV-PAN consists of surgical repair; however, the surgical mortality is high, ranging from $7-20 \%[1,2,5]$. Echocardiography plays a critical role in the assessment of rupture location, orifice geometry, and anatomical relationship with surrounding structures. Although the superiority of real-time threedimensional (3D) echocardiography over twodimensional (2D) echocardiography in the assessment of LV-PAN has been reported, the efficient application of intraoperative real-time 3D transesophageal echocardiography (RT 3D TEE) in the surgical repair of LV-PAN is still unestablished $[6,7]$. We present a case of LV pseudoaneurysm (LV-PAN) formation without a major coronary lesion that developed 1 year after aortic root 
replacement combined with ascending aorta and hemiarch replacement, regardless of no findings of PAN and myocardial ischemia in the perioperative period. Intraoperative real-time three-dimensional transesophageal echocardiography (RT 3-D TEE) during the surgical repair of LV-PAN could contribute to avoiding surgical complications and better understanding of the anatomical structure and information sharing with cardiac surgeons.

\section{Case presentation}

A 56-year-old man presented with shoulder pain at rest and ST-segment elevation in leads II, III, aVF, V5, and V6. He had a history of valve-sparing aortic root replacement (the David procedure) combined with ascending aorta and hemiarch replacement for annuloaortic ectasia 1 year prior to presentation. His LV function immediately after surgery was normal without any regional wall motion abnormality. Coronary angiography was performed under the suspicion of acute coronary syndrome; $90 \%$ stenosis of the diagonal branch of the left anterior descending artery was detected. Transthoracic echocardiography (TTE) revealed a $17-\mathrm{mm}$ perforation in the inferior wall of the LV and a PAN with a diameter of 7 $\mathrm{cm}$. The LV dimensions and wall motion were normal. Mild mitral regurgitation was detected. Similar findings were obtained using left ventriculography and cardiac computed tomography.

Because the patient was hemodynamically stable, with a low dose catecholamine administered soon after admission to the intensive care unit, elective surgical repair was planned due to the risk of redo surgery. However, his LV function and respiratory status rapidly deteriorated. He was intubated and placed on mechanical ventilation on hospital day 3. Intraaortic balloon pumping (IABP) was initiated due to low cardiac output syndrome on hospital day 5 , and continuous hemodiafiltration was started on hospital day 7 due to acute kidney injury. As the orifice and size of the LV-PAN were enlarged, urgent surgical repair was performed on hospital day 11.

General anesthesia was induced and maintained using sevoflurane, propofol, remifentanil, and rocuronium. Hemodynamics was maintained with dopamine at $2 \mu \mathrm{g} /$ $\mathrm{kg} / \mathrm{min}$, dobutamine at $4 \mu \mathrm{g} / \mathrm{kg} / \mathrm{min}$, and milrinone at $0.35 \mu \mathrm{g} / \mathrm{kg} / \mathrm{min}$, all of which had been administered before surgery. Intraoperative two-dimensional (2-D) TEE revealed LV free wall rupture with a large echo-free space in the inferior wall (Fig. 1). The RT 3-D TEE image clearly showed that the orifice of the PAN was located at the mid-inferior segment of the LV (Fig. 2), adjacent to the attachment site of the posteromedial papillary muscle (Fig. 3). The orifice of the PAN was located at the basal side of the insertion of the posteromedial papillary muscle; thus, we informed the cardiac surgeons that the repair procedure might affect the function of the posteromedial papillary muscle. Both side orifice images from PAN cavity (Fig. 3left) and LV cavity (Fig. 3right) allowed the surgeon to better understand the precise structure and plan the appropriate repair. In

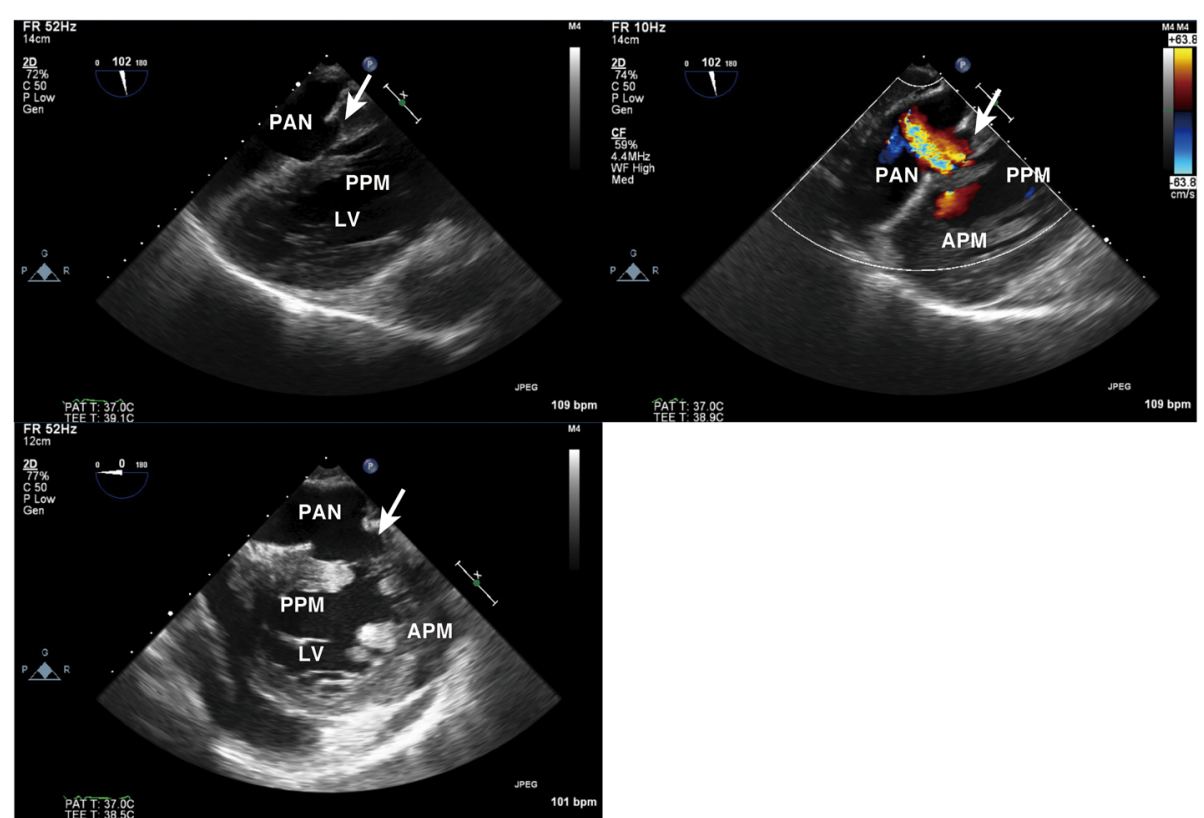

Fig. 1 Transgastric long-axis view (two-dimensional, upper left; color flow Doppler, upper right) and mid-papillary short-axis view (lower). The orifice of the pseudoaneurysm is located in the inferior wall of the left ventricle (arrow). APM anterolateral papillary muscle, LV left ventricle, PAN pseudoaneurysm, PPM posteromedial papillary muscle 


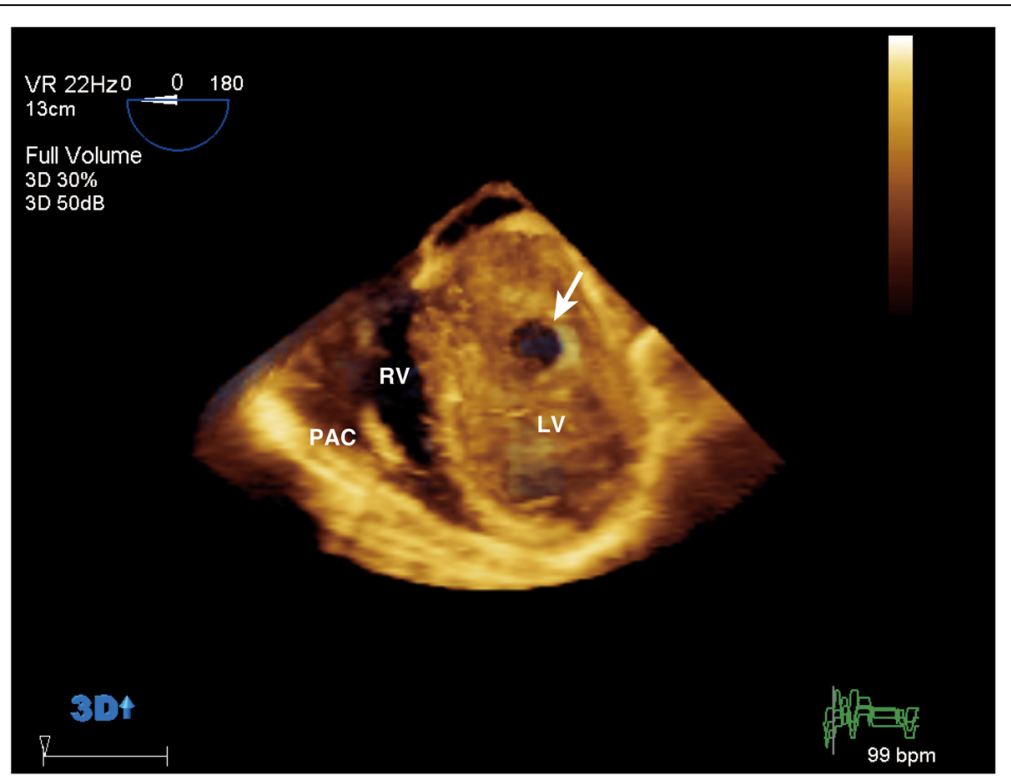

Fig. 2 Three-dimensional view of the orifice of the pseudoaneurysm from inside the LV (arrow). The orifice of the pseudoaneurysm was located in the inferior wall. This three-dimensional view clearly shows the shape of the orifice. LV left ventricle, PAC pulmonary artery catheter, RV right ventricle

particular, 3-D images of the orifice from outside of LV cavity (Fig. 3left), which clearly visualized the posteromedial papillary muscle through the hole, were much useful to decide the suture site. The TEE image also showed minimal functional mitral regurgitation.

After a cardiopulmonary bypass (CPB) was established through femoral arteriovenous cannulation, a median sternotomy was performed, and adhesions were carefully detached to reach the LV-PAN. There was an extensive necrosis of the epicardium and a hematoma between the epicardium and the muscle layer were confirmed. A $2 \times$ 2 - $\mathrm{cm}$ defect of the LV wall formed the orifice of the
PAN. The defect was closed carefully using the double patch technique to avoid injury of the papillary muscle. The patient was smoothly weaned from cardiopulmonary bypass with dopamine at $3 \mu \mathrm{g} / \mathrm{kg} / \mathrm{min}$, dobutamine at $3 \mu \mathrm{g} / \mathrm{kg} / \mathrm{min}$, milrinone at $0.5 \mu \mathrm{g} / \mathrm{kg} / \mathrm{min}$, and IABP. The surgery was completed, with difficulty in achieving hemostasis. TEE after weaning from CPB revealed neither exacerbation of the mitral regurgitation nor any remarkable changes in the morphology of the mitral valve (Figs. 4 and 5). The postoperative course was favorable and the patient was discharged in an ambulatory state on postoperative day 18.

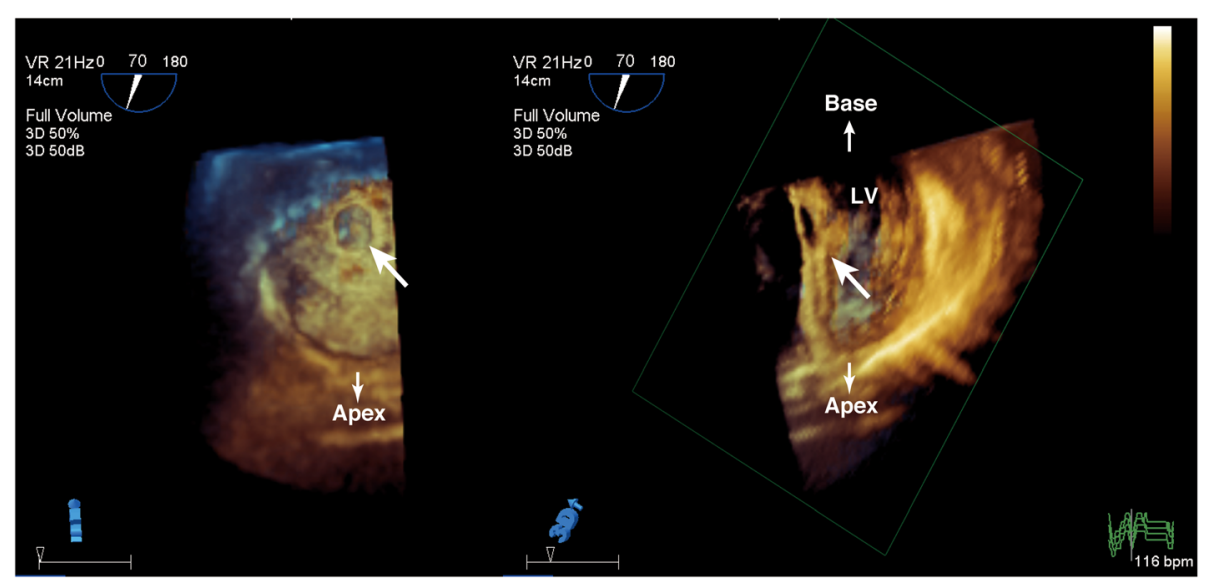

Fig. 3 Three-dimensional images of the orifice of the pseudoaneurysm from outside (left) and inside (right). The images were obtained in the transgastric view. The attachment site of the posteromedial papillary muscle (arrows) is located very close to the orifice. This finding suggests that repair of the left ventricular pseudoaneurysm might influence papillary muscle function 


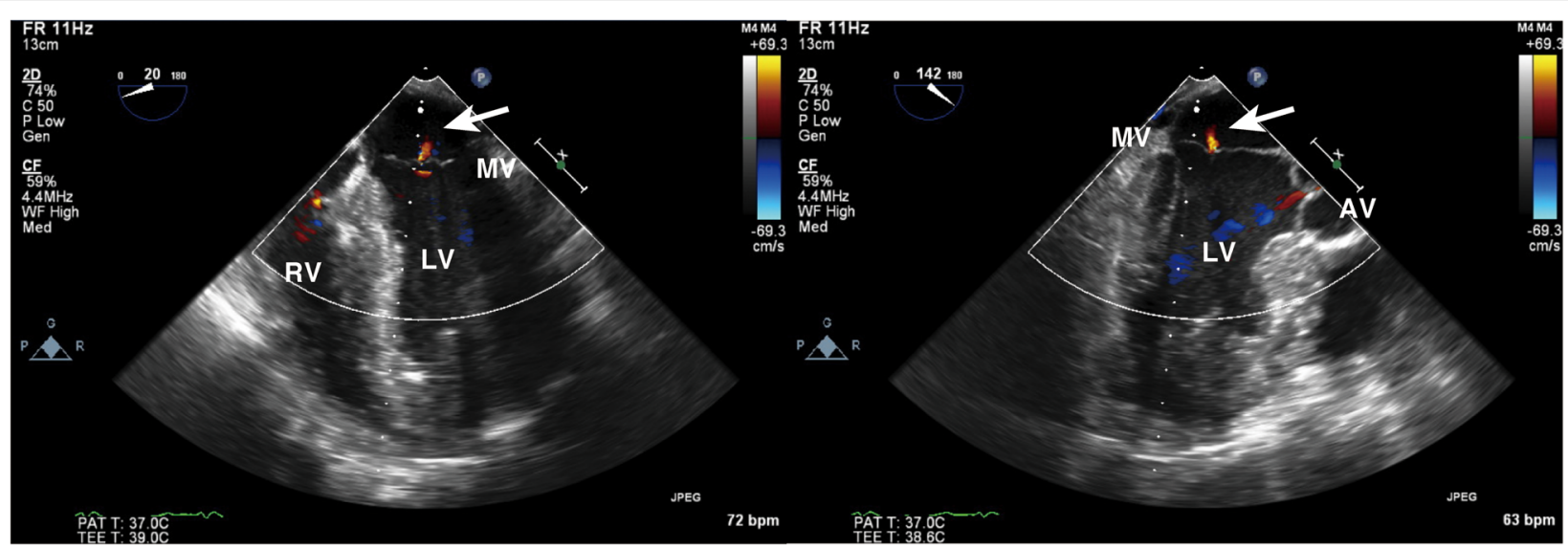

Fig. 4 Color-flow Doppler images of mid-esophageal four chamber view (left) and midesophageal long axis view (right) after weaning from cardiopulmonary bypass. There is trivial mitral regurgitation (arrow) after the repair. AV aortic valve, LV left ventricle, MV mitral valve, RV right ventricle

\section{Discussion}

In the present case, the patient previously underwent aortic root surgery without direct manipulation of the rupture site. There are few reports of cases of LV-PAN formation after aortic root surgery; one reported case described LV-PAN in the LV outflow tract [8]. Injury of the LV by the venting catheter could be a cause of PAN formation; however, the early onset of LV-PAN formation should be expected [9]. Although chronic myocardial ischemia due to the surgical procedure might also be a cause of LV-PAN, no culprit coronary lesion was found and the patient's LV function after the previous surgery was normal without any regional wall motion abnormality. Thus, an obvious cause of LV-PAN formation could not be determined.

Intraoperative RT 3-D TEE could add spatial information to 2-D TEE and demonstrate the proximity of the rupture site to the posteromedial papillary muscle. Some authors reported that 3-D echocardiography could add spatial information, including the location of the LV rupture, orifice geometry, and complex intracardiac flow, in comparison with 2-D echocardiography $[10,11]$. The spatial relationship between the orifice and the papillary muscle is crucial in the surgical repair of LV-PAN, as the repair procedure can induce direct papillary muscle injury and alter the geometry and function of the papillary muscle and mitral valve [12]. The diagnostic modalities for LV-PAN include TTE, TEE, left ventriculography, cardiac computed tomography, and cardiac magnetic resonance imaging [1]. The strengths of RT 3-D TEE compared to the other modalities are better spatial resolution and the ability to easily obtain diverse images useful for the understanding of the anatomical relationship. In addition, 3-D images can provide better sharing of the spatial findings among surgical team members than 2-D imaging because the spatial relationship of structures obtained by 2-D TEE is subject to each observer's perception.

The efficient application of RT 3-D TEE in cardiac surgery is still evolving, and it has been shown useful for

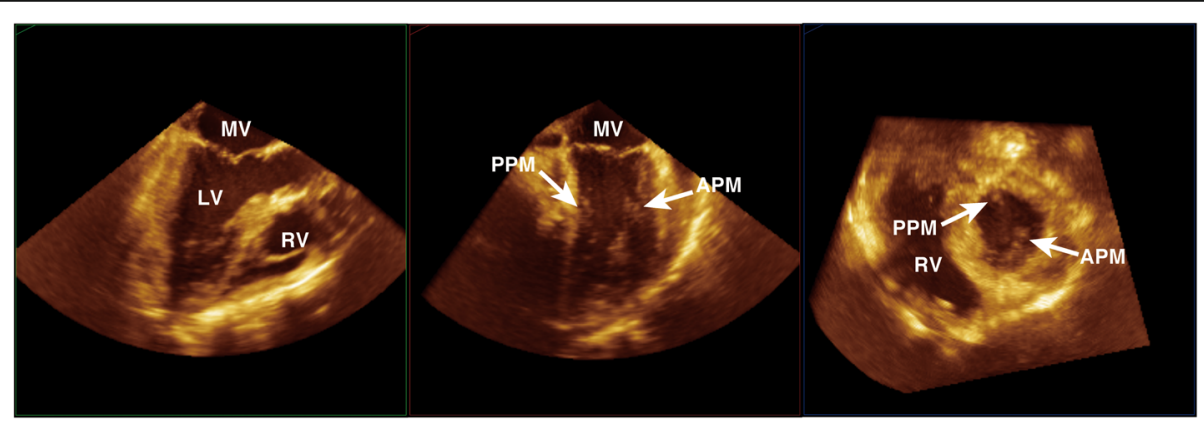

Fig. 5 Mid-esophageal long axis view (left), mid-esophageal mitral commissural view (middle), and short axis view of the left ventricle (right). These images were obtained thorugh multiplanar reconstruction from a volume dataset after weaning from cardiopulmonary bypass. The morphology of the mitral valve and posteromedial papillary muscle is normal. APM anterolateral papillary muscle, LV left ventricle, MV mitral valve, PPM posteromedial papillary muscle RV right ventricle 
the evaluation of the native valves, prosthetic valves, and the left ventricle [13]. Intraoperative RT 3-D TEE can be indicated in cases where the precise understanding of the anatomical structures is required for clinical decision-making and where information sharing among the surgical team is critical for conducting adequate procedures. The en face view and multiplanar reconstruction that RT 3-D TEE provides may be suitable for satisfying the surgical team's needs in such cases.

In conclusion, LV-PAN formation after cardiac surgery can present without direct manipulation of the rupture site and major coronary lesion. Intraoperative RT 3-D TEE can facilitate better understanding of the anatomical relationship between the rupture site and the posteromedial papillary muscle and allow for information sharing to avoid complications during surgical repair.

\section{Abbreviations}

2-D: Two-dimensional; 3-D: Three-dimensional; CPB: Cardiopulmonary bypass; IABP: Intraaortic balloon pumping; LV: Left ventricle; LV-PAN: Left ventricular pseudoaneurysm; PAN: Pseudoaneurysm; RT 3-D TEE: Real-time threedimensional transesophageal echocardiography; TEE: Transesophageal echocardiography; TTE: Transthoracic echocardiography

\section{Acknowledgements}

The authors thank Prof. Takeshi Nomura (Department of Intensive Care Medicine, Tokyo Women's Medical University) for his useful advice on the manuscript.

\section{Authors' contributions}

FM carried out anesthetic management and wrote the manuscript. YS carried out anesthetic management, performed ultrasound imaging, wrote the manuscript, and conducted the literature review. $\mathrm{MN}$ and $\mathrm{MO}$ reviewed and commented on the manuscript. All authors read and approved the final manuscript.

\section{Funding}

This report did not receive any specific grant from funding agencies in the public, commercial, or not-for-profit sectors.

\section{Availability of data and materials}

The data in this case report are available from the corresponding author on reasonable request.

\section{Ethics approval and consent to participate}

Not applicable.

\section{Consent for publication}

Written informed consent was obtained from the patient for publication of this report and any accompanying images.

\section{Competing interests}

The authors declare that they have no competing interests.

Received: 24 April 2019 Accepted: 14 June 2019

Published online: 21 June 2019

\section{References}

1. Frances C, Romero A, Grady D. Left ventricular pseudoaneurysm. J Am Coll Cardiol. 1998:32:557-61.

2. Yeo T, Malouf J, Oh J, Seward J. Clinical profile and outcome in 52 patients with cardiac pseudoaneurysm. Ann Intern Med. 1998;128:299-305.

3. Neupane S, Kommuri NV, Kazanji N, Chowdhury P. Left ventricular pseudoaneurysm after mitral valve replacement. Echocardiography. 2016;33: 1788-9.
4. Baumann S, Renker M, Spearman JV, Bayer RR, Schoepf UJ, De Cecco CN. Giant left ventricular pseudoaneurysm as a complication after mitral valve replacement surgery. Ann Thorac Surg. 2014;98:1480.

5. Atik FA, Navia JL, Vega PR, Gonzalez-Stawinski GV, Alster JM, Gillinov AM, et al. Surgical treatment of postinfarction left ventricular pseudoaneurysm. Ann Thorac Surg. 2007;83:526-31.

6. Nekkanti R, Nanda NC, Zoghbi GJ, Mukhtar O, McGiffin DC. Transesophageal three-dimensional echocardiographic assessment of left ventricular pseudoaneurysm. Echocardiography. 2002;19:169-72.

7. Arat N, Ozbulbul NI. Three-dimensional echocardiography is a useful tool in the diagnosis of left ventricular pseudoaneurysm. Eur J Cardiothorac Surg. 2007;32:164.

8. Inoue T, Hashimoto K, Sakamoto Y, Yoshitake M, Matsumura Y, Kinami H, et al. Left ventricular pseudoaneurysm after reimplantation procedure. Gen Thorac Cardiovasc Surg. 2016;64:224-6.

9. Arslan S, Erkut B, Ates A, Kantarci M, Kalkan ME, Karakelleoglu S. Pseudoaneurysm of left ventricular following left ventricular apical venting. Clin Res Cardiol. 2009;98:280-2

10. Little SH, Ramasubbu K, Zoghbi WA. Real-time 3-dimensional echocardiography demonstrates size and extent of acute left ventricular free wall rupture. J Am Soc Echocardiogr. 2007;20:538.e531-3.

11. Avsar S, Keskin M, Velibey Y, Kemaloglu Oz T, Guvenc TS, Guzelburc O, et al. Incremental utility of real time three-dimensional transthoracic echocardiography for the assessment of left ventricular free wall rupture location, orifice geometry, and complex intracardiac flow. Echocardiography. 2015;32:1738-41.

12. Essandoh M, Otey AJ, Crestanello J. Severe mitral regurgitation after left ventricular pseudoaneurysm repair. J Cardiothorac Vasc Anesth. 2015;29: 1295-300.

13. Mahmood F, Jeganathan J, Saraf R, Shahul S, Swaminathan M, Mackensen $\mathrm{GB}$, et al. A practical approach to an intraoperative three-dimensional transesophageal echocardiography examination. J Cardiothorac Vasc Anesth. 2016;30:470-90.

\section{Publisher's Note}

Springer Nature remains neutral with regard to jurisdictional claims in published maps and institutional affiliations.

\section{Submit your manuscript to a SpringerOpen ${ }^{\circ}$ journal and benefit from:}

- Convenient online submission

- Rigorous peer review

- Open access: articles freely available online

- High visibility within the field

- Retaining the copyright to your article

Submit your next manuscript at $\boldsymbol{\nabla}$ springeropen.com 